It was with the hope of avoiding these complications that I was leil, now more than two years agro, to try the plan of subcutaneous division of the veins by means of a platinum wire heated to a red heat by electricity. The procedure is, as you have seen, very simple. First, feel for the vas deferens, and grasp the veins in front of it, and nip in the scrotum with the left thumb and forefinger; transfix the scrotum at this spot between the duct and the veins with a narrow bistoury, and pass a needle armed with a platinum wire in the track of the knife; then return the needle through the same apertures, but this time in front of the veins between them and the skin. Of course, if the vas happen to be in front of the veins, as was the case in that of W. C- , shown to-day, you modify the procedure a little. In this way you have the veins in a loop of the wire. It is better to make a puncture with a knife rather than merely to transfix with the needle, for the veins are looped up cleaner, there is not the same liability to include a portion of the skin in the noose as in the latter plan. Then attach the ends of the wire to the écraseur, and connect with the battery, using sufficient cells to cause a faint hissing noise ; one cell of Grove's battery, or at most two, is sufficient. This step must be done deliberately; I have taken as long as five or six minutes over it. To protect the skin from burning, let some cold water trickle over it while the wire is burning its way through the veins. The after-treatment consists in perfect rest in bed for a few days, with the scrotum supported on a broad strip of strapping fixed across the front of the thighs. I have three times operated without anæsthesia, but the pain may be severe, and I prefer to have the patient under the influence of ether. After recovery from the anæsthesia there is an entire absence of pain, and this perfect freedom from spontaneous pain continues uninterrupted throughout the convalescence. A few hours after the operation the knobby feel of the varix is replaced by a soft even swelling, which lessens and hardens, and at the end of forty-eight hours is usually to be felt as a hard lump, about the size of a big marble. This is tender on pressure. By the end of a week it has lost its tenderness, and has shrunk to three-quarters its original size. The veins below can still be felt full, but not compressible, the blood in them has by that time coagulated, and they become smaller and firmer, until ultimately a small pea-like induration in front of the vas is all that is left, and even this may disappear, and no trace of the varix or operation be left, as in W. $\mathrm{S}$ -

I have employed this method eight times, each time the varix has been cured, the symptoms complained of relieved, and no mischief to the testicle has followed. These eight cases have shown me that there are three things to be carefully avoided. The first is hurry in the section of the veins; twice a few drops of blood have followed the escape of the wire, but only so little as to require nothing more than lint or cotton-wool to be placed over the wound ; in neither instance was there any hæmatocele. The second is to operate well above the tunica vaginalis; in one of my cases acute hydrocele was lighted up, it quickly subsided under belladonna fomentations. The third is burning of the skin, leading to a small slough; although there is nothing of serious moment in this slough, it is of course best to avoid it, and if the skin is incised and kept cold and the loop of wire not brought too small it can generally be saved.

I can recommend you to practise this operation, first, because of its entire painlessness after the few moments of its actual performance, and, secondly, because it fulfils the physiological indications better than any other I know of. My cases at present are too few to prove by statistics that it is less dangerous to life than those operations I have mentioned, but they are sufficient to show that the operation is simple, effectual, and introduces no new danger, while it is obviously free from the sources of irritation common to other methods.

The section of the veins is completed at once ; there is no foreign body liable to set up inflammation left in contact with the healing veins, which are allowed to remain at perfect rest, while their seared ends are becoming permanently closed. As you are aware, the closure of an open vessel by the hot wire or iron is usually considered a particularly safe plan. There is also no interference with the skin of the scrotum, as in Lee's operation; no compression of the scrotal vessels, no cdema.

The only other method that I should at all recommend to you is the use of the catgut ligature applied to the veins subcataneously. I have had no experience in it, but it has been done with success. I think the heated wire is preferable, because the catgut ligature is rather uncertain in its action; it may soften very rapidly and then fail to occlude the vessels, or it may not soften at all and cut it way through the veins like an ordinary hempen or silk thread, or remain and subsequently light up inflammation. These accidents are only occasional, but we should none the less be on our guard against them, and give the preference to a plan of treatment which is free from them. In none of my eight cases has any slough separated from the veius the eschar formed has been absorbed by the living tissues. I show you to-day four cases at varying periods after the operation illustrating their progress.

1. W. J-, aged seventeen, a large left varicocele, which he had noticed for " several years," left testicle smalle than right, complained of dragging pains in scrotum and up to left loin. Operation June 21st, 1879 ; left hospital June 28th. A firm induration the size of a marble felt; not tender ; veins below plugged.

2. F. L_- aged twenty-four, single, injured left testicle five years ago; first noticed varicocele three years ago, after an attack of clap, since then the pain and swelling had steadily increased in spite of wearing a suspender. No constipation; external piles for two years. Operation May 28 th ; left hospital June 8th. The induration is very hard, the size of a horse bean; veins below are firm and shrinking.

3. W. C-- , aged thirty, single, very large left varicocele, noticed suddeuly in June, 1879 , while bathing. Pain in scrotum and groin had increased of late; testicle smaller than right. Operation May lst; left hospital May 7th; delayed on account of a slough of skin. Induration now small ; veins below cannot be felt; testicle has regained its former size and is a little larger than right.

4. W. S_- twenty-one, a small varicocele noticed some years ; scrotum not lax ; considerable pain ; frequent semi. nal emissions and mental depression. Operation Sept. 29th; left hospital Oct. 9th. No trace of dilated veins or of the seat of the operation to be felt. All symptoms relieved.

\section{SOME STATISTICS OF FATAL CHOREA.}

By OCTAVIUS STURGES, M.D.,

PHYSICIAN TO THE WESTMINSTER HOSPITAL ; ASSISTANT PHYSICLAN To THE HOSPITAL FOR SICK CHILDREN.

The pathology of chorea needs to be discussed upon a wider basis than that which is offered by morbid anatomy. Nevertheless, no survey of the disorder can be complete which fails to take into account evidence of this sort. The review of a sufficient number of examples of fatal chorea, apart from any direct discovery of morbid change, cannot fail to be of service in exhibiting the age and sex and tem. perament most liable to succumb to such attacks. We are thus assisted in separating from one another a highly dangerous form of convulsion, and that far commoner motor disorder which, whether violent or not, has little or nothing of danger about it, and probably recovers best without active medical interference.

Yet, with this obvious service to be got from the study of fatal chorea, the event of death is so rare that no single observer from his own experience can do more than contribute a very small share of the material necessary for the purpose of drawing any general conclusions. Thus, Dr. Hughes, in so large a hospital as Guy's, was able to collect from all the records by various hands which had been preserved during more than thirty years, only eleven fatal cases. Dr. Dickinson, from a similar record at St. George's, extending over thirty-three years, collected sixteen such cases, while the large field of observation offered by the Hospital for Sick Children during fifteen years furnishes but six. These examples, it must be understood include those dying with chorea as well as those dying of it. Although it is not always easy to distinguish between these two classes, it is certain, as will presently appear, that the cases of death attributable directly to chorea fall very far short of the numbers just given. It must be added that many of the earlier records are very defective, that there is no uniform plan of tabulation, and that with different observers different points of interest have received mention. 
On the whole it may be said that if all the recorded accounts of fatal chorea in this country which are fairly complete and readily accessible were put together the total number would not be large, and that in a considerable proportion of these the immediate cause of death and the choreic disorder would be found in very remote and uncertain connexion.

Such being the facts of the case, I have thought it of interest to bring together eighty cases of death in connexion with chorea, or at least with convulsions resembling and called chorea. They are made up as follows:-(a) Three cases (quoted by Dr. Bright), Med. Clin. Trans, 1839 ; (b) eleven cases (Dr. Hughes), Guy's Hospital Reports, 1846 (first series) ; (c) seven cases (from same source), 1855 (second series) ; $(d)$ thirty-four cases (Dr. Tuckwell), Bartholomew's Hospital Reports, vol. v.; (e) twenty-two cases (Dr. Dickinson), Med. Clin. Trans., vol. lix.; $(f)$ three cases (Dr. Peacock), St. Thomas's Hospital Reports, vol. viii.

With these references and the frequent mention $I$ shall have to make of each of these eminent authors no one can quote against me on their behalf the line that Virgil wrote on the palace gate. The present object is to bring together under one point of view a body of evidence derived from many sources without admixture of extraneous matter. Of the conclusions to be drawn from such evidence the reader will judge for himself.

The points I propose to examine concern the age and sex and common exciting cause of fatal chorea, together with its most obvious morbid associations. It will be seen immediately that only a proportion of the eighty cases are given in such detail as to be dealt with in all these respects. Thus Dr. 'Tuckwell's account is no more than a short summary occupying one paragraph of his paper just quoted. Dr. Hughes's first series is described by himself as "very meagre and imperfect;" Dr. Bright's three cases, although so of ten quoted, are not altogether in point; in one there was trismus and tetanic spasm, and in a second paroxysmal attacks described as hysteric. In the several enumerations now to be made, therefore, while eighty will be the extreme number dealt with, smaller numbers will have to serve in regard to particular points where the information is defective. With such deduction the cases available in all respects will not exceed thirty, but thirty in the circumstances of this disorder is a large number, it is the gleaning of a very wide field, the sum of fatality out of many thousand examples of chorea extending over a long period of years.

Taking in the first instance the gross number eighty as representing so many individuals dying in connexion with chorea, the most striking point of all to those who are in the habit of regarding chorea as a child's affection is the large proportion of adults and the small proportion of children. Of the eighty, forty-eight are over thirteen years of age, and the ages of two are not stated. Excluding Dr. Tuckwell's numbers, where further particulars are not given, we have in forty-six fatal cases twenty-seven who are over thirteen and nineteen who are under that age. Of the twenty-seven over thirteen, as many as twenty-three may be said to have died of chorea. Of the nineteen children, ten at the utmost died of it, the youngest of these being a girl of seven (No.19 in Dr. Dickinson's table, loc. cit.). Or, again, excluding Dr. Hughes's first (and defective) series, we should have, out of a total of thirty-five, twenty fatal cases over the age of thirteen, of whom sixteen died apparently of chorea ; and fifteen uuder thirteen, of whom only seven died apparently of chorea. We thus establish the fact, making large allowance for error (which from the nature of the material cannot be wholly excluded), that the mortality of children directly from chorea is exceedingly small, and that seven is the youngest age at which it is known as a fatal disease in a very extensive review. We find also, as we should expect in view of the comparative commonness of chorea in children, that the proportion dying with chorea but not of it is greater with them than with adults.

But next in regard to sex. In forty-six fatal cases (again excluding Dr. Tuckwell's) thirty-four are females and twelve males. Of the thirty-four females eight only were children, the youngest (mentioned above) seven years old, and one described only as "a child." As many as twenty-six were young women, eleven of whom, there is good evidence to show, died of chorea. Of the twelve males nine were adults and only three young boys. These latter are made up as follows: One is in Dr. Hughes's first series (No. 6), one is in Dr. Dickinson's table (No. 14), and died of heart affection "without return of chorea," and one is a case of the late Dr. Fuller's (No. 9 in the same table) that died under my own observation as then Medical Registrar at St. George's Hospital, after five months of almost persistent convulsion. These three boys were each eleven years old. It does not appear upon the evidence before us that chorea is ever fatal to males at an earlier age than this, nor have we more than one out of the three whose death is immediately attributable to this disorder.

The puberty age, then, is the age of fatal chorea. If we take forty-six as representing the total number of persons dying in this connexion, thirty-five would be at or a little above the age of puberty, and twenty-five of these young women. ${ }^{1}$ We are thus led to associate fatal chorea with the disturbing incidents of a critical period of life. And the more so inasmuch as in those instances where exciting causes are actually discovered and recorded (but a small proportion, it must be remembered, of the whole number), there is frequent mention of some special excitement of those parts and organs which in the plan of nature are properly over-active at this particular time. Thus Dr. Hughes, in his second series of cases, finds the genital organs unusually excited in three of the four cases examined, while in Dr. Dickinson's table out of nine dying directly of chorea, and with causes assigned, there are three in whom these causes are distinctly sexual.

And not only does fatal chorea attach peculiarly to the emotional sex and the emotional time of life, there are but few instances of it out of the few fully detailed cases which constitute our entire material in this matter where mental excitement does not concur with the bodily over-movement. Such mental disturbance embraces all varieties, from the hysterical to the maniacal. Here again, therefore, a distinction appears between the fatal chorea of adolescence and the non-fatal chorea of childhood. The latter, even in its greatest violence, is commonly quite apart from emotion. The disorder acquires this mental admixture at the same time that it reaches what may be called its dangerous age. It will be perhaps better in this place to cite instances rather than to quote figures. The condition to which $I$ am alluding is manifested by particular incidents. Thus in a case related by Dr. Hughes (first series, loc. cit., p. 390) a man of twenty-five, with violent and ultimately fatal chorea, was "sensibly hurt and shed tears when the bystanders observed that he could restrain the movements if he pleased." In a second case, by the same author (second series, p. 250), that of a girl of eighteen, the symptoms "which were at first slight were suddenly aggravated by fright caused by a patient in the same ward, and she became almost maniacal." In a third instance (from the same series, p. 25l), a girl of sixteen, where "there was unusual excitement of the generative organs," the symptoms at first "partook of the character of hysterias, the patient appeared strange in her movements, and fancied that others were laughing at her." In a fourth, a boy of sixteen, "supposed to be addicted to secret vice, his early movements excited the derision of his comrades. Their conduct depressed him much, and he went suddenly into a fit, after which violent and universal chorea supervened." In all three of Dr. Peacock's fatal cases mental excitement is a prominent feature. In one of the very few cases that are recorded of chorea fatal as early as ten (Dr. Dickinson, loc. cit., p. 2), it is related of the child (and some will probably regard the relation as trivial) that a little friend of hers had lately died of chorea, she had watched and imitated the movements, and when she recognised them in her own person made sure that she should meet the same fate. If it be remembered from how small a number of examples these instances are taken, even these few quotations will be allowed to have weight in showing the common habit of fatal chorea. I think it may be said, indeed, not only of fatal chorea but of chorea that at any part of its course threatens to be fatal (a comparatively large number) that amongst the symptoms that give alarm nervous exaltation (so to speak) is generally one.

But while the emotional origin of chorea is thus apparent, as well as the direct influence of mental causes in determining its fatal issue, it is not to be doubted that fatal chorea arises in some instances in immediate connexion with acute rheumatism. The evidence upon this point now before us is to the following effect:- Out of thirty-two fatal cases dying with chorea, and reported with sufficient fulness to be available (viz, seven of Dr. Hughes', second series, twenty-two of Dr. Dickinson's, and three reported by Dr.

1 There is but one elderly person on the list, a woman aged fifty-four, who had had chorea for four years. (Dr. Dickinson, loc. cit., No. 15.) 
Peacock, seven had a rheumatic origin. Selecting out of the thirty-two eighteen who died directly of chorea (viz., five of Dr. Hughes', ten of Dr. Dickinson's, and all three of Dr. Peacock's), we get three-a male and two females, all adults-where the fatal disorder was of rheumatic origin. These numbers corroborate what I have before maintained in this journal-viz., that acute rheumatism, al though having but a sinall share in chorea, is nevertheless veritably associated with it, and especially with its fatal form at the puberty age.

Coming now to the morbid associations of chorea in so far as they are revealed post-mortem, the evidence hefore us fully hears out the belief expressed by the late Dr. Kirkes in 1863 , "that further experience would still more positively demonstrate that an affection of the left valves of the heart with the presence of granular vegetations upon them is an almost invariable attendant upon chorea, under whatever circumstances the chorea may be developed." The singular accuracy of this description, the need of "almost," and the justice of applying the observation to chorea of whatever origin, and not limiting it (as some would do even now) to rheumatic chorea, or even to chorea fatal as such, will be seen in what is now to be said. Out of all the cases in which the condition in question is especially mentioned I can find but five where the heart valves and pericardium are reported healthy, one of these being an elderly woman. Wherever the heart is affected the mitral valve is affected; the aortic valves are implicated often, the pericardium sometimes. This heart affection occurs equally in those that die in connexion with chorea, and in those that die directly of it. Thus, of Dr. Dickinson's twenty-two there are, as I reckon, twelve dying with, but not of, chorea; of these ten have mitral valve vegetations, and two have not. Of the ten dying of chorea seven have vegetations, and three have not. In Dr. Peacock's three cases, all dying of chorea, two have vegetations, and one has not. The valve deposit, which for shortness I thus name, may be either old or new, and variously connected with the mitral valves on their auricular side. The condition would seem to be an accident of the disease it accompanies, having but little apparent connexion with its fatal result. It so happens that of the five cases just mentioned as being the only examples of chorea occurring with perfectly healthy heart, all save one, the old woman, are typical instances of deaths in and by chorea. Three were girls at about the puberty age (Nos. 1, 5, and 8 in Dr. Dickinson's table), and one is a girl of Dr. Peacock's, aged twelve (St. Thomas's Reports, vol. viii., p. 29).

With the exception of this observation in regard to the heart and pericardium, there is little to be gathered in the material now before us as to the morbid anatomy of fatal chorea. I need not now refer to Dr. Dickinson's investiga. tions as to the minute nervous changes in seven of the cases here mentioned, nor to those of Dr. Tuckwell upon embolic plugging, in the paper above quoted. Speaking generally, it must be said that the state of the brain and cord is not described in sufficient detail to be instructive. In a large proportion the brain, in a few the cord, is described as "congested." In the case of the boy to whom I have alluded as the single example of the kind in my own experience or in these tables, it was observed that " the grey matter of the cord was altered and yellow in patches," but there was no minute examination.

How far the foregoing particulars may serve to fortify or disturb pathological doctrines I do not now inquire. Their practical teaching is obvious. We are justified upon this evidence in looking upon severe chorea at the puberty age, especially in connexion with sexual excitement or with acute rheumatism, as a dangerous disorder apt to terminate fatally. It is true that we do not number many fatal cases, but it is also true that we do not meet with many, whether fatal or otherwise. The condition of such patients is the more precarious when, as is commonly the case, there is emotional as well as motor disorder. Such nervous exaltation, which is commonly altogether absent in the more faniliar chorea of childhood, is with these both the sign and the source of danger. They are in a state of extreme sensitiveness, and lay hold of trivial circumstances to aggravate their disquiet. The smallest incident, the conduct of bystanders, undeserved reproach or ridicule, or even the sight of a patient affected like themselves, will often have its immediate prejudicial influence in a degree which those who have little experience of this kind of convulsion (whose actual title to the name of chorea, as Dr. Bright's cases show, is often far from obvious) will find it difficult to believe. The question arises whether in these circumstances we address our treatment sufficiently to this mental state.

But the facts before us have this further teaching. If we exclude puberty, chorea of whatever violence is hardly dangerous to life. If we exclude both puberty and the female sex, chorea of whatever violence is not dangerous at all. Now violent chorea is by no means uncommon with boys. Thousands of examples of it must be comprehended within the period we are now reviewing. And yet in all that time, and from so many fields of observations, we get but one boy, aged eleven, dying of chronic chorea after tive months, and probably with sclerosis of the cord. Chorea therefore, we may say, extremely rarely fatal in little girls, is, practically speaking, never fatal in little boys. If we add to this, what will not be denied, that permanent dis. ablement from chorea is very uncommon, and that there is no treatment that even pretends to avert such after-effects, we get a strong argument in favour of leaving childish chorea alone. This conclusion will be further strengthened by a perusal of the valuable papers published in THE LANCET of Nov. 18th, 1876, by Drs. Tuckwell and Gray, upon "The Expectant Treatment of Chorea."

The conclusions to be derived from the foregoing review may be expressed as follows :-

1. Chorea, regarded as a disease of itself fatal, belongs almost exclusively to puberty, and especially to female puberty ; its immediate exciting cause having distinct re. ference, in many instances, to conditions of unusual sexual excitement.

2 . Besides the operation of sexual causes mental disturb. ance has to be reckoned; not fright only, but worry, anxiety, and despondency also, while the force and in fluence of such impressions is to be seen in the course as well as in the origin of fatal chorea.

3. Acute rheumatism appears as a cause of fatal chorea in but a small proportion of cases; yet the association, in frequent as it is, is distinct and unquestionable.

4. Chorea in its fatal, no less than in its non-fatal forms, shows strong preference for the female sex at all ages. Children, however, very rarely die of it, and boys, practically spoaking, never.

5. Mental excitement, in varying degree (although not amongst the symptoms of ordinary chorea), is met with in so large a proportion of its fatal examples that we are justified in regarding this concurrence as of bad augury.

6. "Vegetations," new or old, on the auricular surface of the mitral valves, with or without similar deposit on the aortic valves, and sometimes with pericarditis, are met with in the great majority of cases dying of, or with, or shortly after, chorea. This condition, however, does not, as a rule, contribute directly to the fatal issue; it is found equally amongst those that die with and those that die of chorea. In some of the most marked and typical cases of fatal chorea the valves of the heart have been found absolutely healthy.

7. There is no other morbid condition, except that which concerns the heart, occurring with sufficient frequency or uniformity to be regarded as characteristic of fatal chorea.

Wimpole-street.

\section{OBSERVATIONS IN URINARY PATHOLOGY AND THERAPEUTICS.}

By C. H. RALFE, M.D. Cantab., F.R.C.P. Lond, ASSISTANT PHYSICIAN TO THE LONDON HOSPITAL, SENIOR PHYSICIAX TO THE SEAMEN'S HOSPITAL, LATE TEACHER OF PHYSIOLOGICAI CHEMISTRY IN THE MEDICAL SCHOOL OF ST. GEORGE'S HOSPITAL.

\section{A FORM OF DYSPEPSIA USUALLY ASSOCIATED WITH AY ALKALINE CONDITION OF THE URINE.}

WHEN human urine becomes alkaline it is due to one or other of the following conditions: 1 . To excess of the alkaline carbonates of potash and soda; a condition that frequently occurs normally after the ingestion in large quan. tities of vegetables and fruits. 2. Excessive elimination of the phosphates of the alkaline and earthy bases under conditions that have not been yet thoroughly studied, but which are not unfrequently found preceding or even accompanying saccharine diabetes; or sometimes, as has recently 